\title{
A novel mutation in BCS1L associated with deafness, tubulopathy, growth retardation and microcephaly
}

Jackson, C. B.

2016-04

Jackson , C B , Bauer , M F , Schaller , A , Kotzaeridou , U , Ferrarini , A , Hahn , D , Chehade , H, Barbey , F , Tran , C, Gallati , S, Haeberli , A, Eggimann , S , Bonafe , L \& Nuoffer , J-M 2016, ' A novel mutation in BCS1L associated with deafness, tubulopathy, growth retardation and microcephaly ' , European journal of pediatrics, vol. 175 , no. 4 , pp. 517-525 . https://doi.org/10.1007/s00431-015-2661-y

http://hdl.handle.net/10138/223931

https://doi.org/10.1007/s00431-015-2661-y

publishedVersion

Downloaded from Helda, University of Helsinki institutional repository.

This is an electronic reprint of the original article.

This reprint may differ from the original in pagination and typographic detail.

Please cite the original version. 


\title{
A novel mutation in $B C S 1 L$ associated with deafness, tubulopathy, growth retardation and microcephaly
}

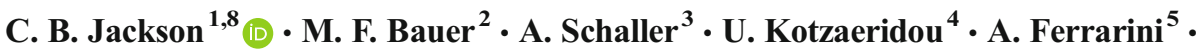 \\ D. Hahn ${ }^{1} \cdot$ H. Chehade ${ }^{6} \cdot$ F. Barbey ${ }^{7}$ - C. Tran $^{7} \cdot$ S. Gallati ${ }^{3}$. \\ A. Haeberli ${ }^{1}$ - S. Eggimann ${ }^{1}$ - L. Bonafé ${ }^{7}$. J-M. Nuoffer ${ }^{1}$
}

Received: 12 June 2015 /Revised: 27 August 2015 / Accepted: 26 October 2015 / Published online: 13 November 2015

(C) Springer-Verlag Berlin Heidelberg 2015

\begin{abstract}
We report a novel homozygous missense mutation in the ubiquinol-cytochrome c reductase synthesis-like $(B C S 1 L)$ gene in two consanguineous Turkish families associated with deafness, Fanconi syndrome (tubulopathy), microcephaly, mental and growth retardation. All three patients presented with transitory metabolic acidosis in the neonatal period
\end{abstract}

Communicated by Jaan Toelen

L. Bonafé and J-M. Nuoffer contributed equally to this work.

Electronic supplementary material The online version of this article (doi:10.1007/s00431-015-2661-y) contains supplementary material, which is available to authorized users.

C. B. Jackson

christopher.jackson@helsinki.fi

M. F. Bauer

bauermat@klilu.de

A. Schaller

Andre.Schaller@insel.ch

U. Kotzaeridou

Urania.Kotzaeridou@med.uni-heidelberg.de

A. Ferrarini

Alessandra.Ferrarini@eoc.ch

D. Hahn

Dagmar.Hahn@insel.ch

H. Chehade

Hassib.Chehade@chuv.ch

F. Barbey

Frederic.Barbey@chuv.ch

C. Tran

Christel.Tran@chuv.ch

S. Gallati

Sabina.Gallati@insel.ch and development of persistent renal de Toni-Debré-Fanconitype tubulopathy, with subsequent rachitis, short stature, microcephaly, sensorineural hearing impairment, mild mental retardation and liver dysfunction. The novel missense mutation c. $142 \mathrm{~A}>\mathrm{G}$ (p.M48V) in BCS1L is located at a highly conserved region associated with sorting to the mitochondria.
A. Haeberli
Annemarie.Haeberli@insel.ch
S. Eggimann
Sandra.eggimann@insel.ch
L. Bonafé
Luisa.Bonafe@chuv.ch
J-M. Nuoffer
Jean-Marc.Nuoffer@insel.ch

1 Institute of Clinical Chemistry, University Hospital Berne, Berne, Switzerland

2 Klinikum der Stadt Ludwigshafen, Ludwigshafen, Germany

3 Division of Human Genetics, Berne, University Hospital Berne, Berne, Switzerland

4 Division of Pediatrics, Heidelberg, Germany

5 Ospedale Regionale di Bellinzona, Bellinzona, Switzerland

6 Pediatric Nephrology Unit, CHUV, Lausanne, Switzerland

7 Center for Molecular Diseases, Lausanne University Hospital, Lausanne, Switzerland

8 Present address: Research Program for Molecular Neurology, Biomedicum Helsinki, University of Helsinki, Helsinki, Finland 
Biochemical analysis revealed an isolated complex III deficiency in skeletal muscle not detected in fibroblasts. Native polyacrylamide gel electrophoresis (PAGE) revealed normal super complex formation, but a shift in mobility of complex III most likely caused by the absence of the BCS1L-mediated insertion of Rieske Fe/S protein into complex III. These findings expand the phenotypic spectrum of $B C S 1 L$ mutations, highlight the importance of biochemical analysis of different primary affected tissue and underline that neonatal lactic acidosis with multiorgan involvement may resolve after the newborn period with a relatively spared neurological outcome and survival into adulthood.

Conclusion: Mutation screening for BCS1L should be considered in the differential diagnosis of severe (proximal) tubulopathy in the newborn period.

\section{What is Known:}

- Mutations in BCSIL cause mitochondrial complex III deficiencies.

- Phenotypic presentations of defective BCS1L range from Bjornstad to neonatal GRACILE syndrome.

\section{What is New:}

- Description of a novel homozygous mutation in BCSIL with transient neonatal acidosis and persistent de Toni-Debré-Fanconi-type tubulopathy.

- The long survival of patients with phenotypic presentation of severe complex III deficiency is uncommon.

Keywords BCS1L · Isolated complex III deficiency and assembly $\cdot$ Mitochondrial disorder $\cdot$ Rieske iron-sulphur protein · Hypoglycaemia $\cdot$ Glycosuria $\cdot$ Deafness $\cdot$ Growth retardation $\cdot$ Fanconi syndrome $\cdot$ Microcephaly $\cdot$ Lactic acidosis

$\begin{array}{ll}\text { Abbreviations } \\ \text { BCS1L } & \begin{array}{l}\text { Ubiquinol-cytochrome c reductase synthesis- } \\ \text { like gene }\end{array} \\ \text { CS } & \begin{array}{l}\text { Citrate synthase } \\ \text { GRACILE }\end{array} \\ & \begin{array}{l}\text { Growth retardation, aminoaciduria, cholestasis, } \\ \text { iron overload, lactic acidosis and early death } \\ \text { syndrome }\end{array} \\ \text { OXPHOS } & \begin{array}{l}\text { Oxidative phosphorylation } \\ \text { Respiratory chain }\end{array}\end{array}$

\section{Introduction}

Mitochondrial disorders are the most common group of inborn errors of metabolism with a prevalence of 1 in 5000 live births [21]. Most mitochondrial disorders directly or indirectly affect energy metabolism, resulting in oxidative phosphorylation (OXPHOS) deficiency. From the five multi-subunit OXPHOS complexes, isolated complex III deficiency is relatively rare [2].
Complex III (coenzyme Q:cytochrome c oxidoreductase, E.C.1.10.2.2), consists of 11 subunits, of which only MTCYB (MIM \#516020) is encoded by the mitochondrial genome, the remainder by the nuclear genome (Supplementary Table S2). Known complex III deficiencies are caused by several nuclear-encoded genes BCS1L (Online Mendelian Inheritance in Man (OMIM) \#603647), CYC1 (OMIM \#615453), LYRM7 (OMIM \#615831), TTC19 (OMIM \#615157), UQCC2 (OMIM \#614461), UQCC3 (OMIM \#616097), UQCRB (OMIM \#615158), UQCRC2 (OMIM \#615160), UQCRQ (OMIM \#615159) and mitochondrial DNA-encoded MT-CYB [22]. Along these genes, which are complex III subunits or assembly factors, known as causes of complex III deficiency (Table S3), more assembly factors are presumed to be identified [11, 12, 15, 25, 26, 29]. Most commonly, mutations in MT-CYB and BCSIL cause (isolated) mitochondrial complex III deficiency. The BCS1 (yeast homolog)like (BCSIL) gene (a member of the AAA family of ATPases) has seven exons, is located on chromosome 2 (2q35) and encodes for a 419-amino acid-long protein localised to the mitochondria [22]. BCS1L acts as a chaperone/translocase in the inner mitochondrial membrane, where it is thought to be a necessary factor to facilitate the insertion of the Rieske $\mathrm{Fe} / \mathrm{S}$ protein for the final assembly step of complex III [4, 28].

Mutations in $B C S 1 L$ are associated with i) Bjornstad syndrome (OMIM \#262000) characterised by sensorineural hearing loss and abnormal flat twisted hair shafts (pili torti); ii) a lethal neonatal metabolic syndrome characterised by foetal growth retardation, aminoaciduria, cholestasis, iron overload, lactic acidosis and early death (GRACILE syndrome: OMIM \#603358); and iii) complex III deficiency (OMIM \#124000) presenting with encephalopathy of variable severity, tubulopathy and/or hepatomegaly [7, 13, 27]. Whereas Bjornstad syndrome is relatively mild, GRACILE syndrome was first described in 1998 in 17 Finnish children presenting with severe lactic acidosis from birth on leading to death within 4 months of age [7]. The renal Fanconi-type tubulopathy with aminoaciduria, glycosuria, phosphate and bicarbonate leak, together with various degrees of growth retardation, microcephaly and deafness, are on the other side common features of isolated complex III deficiency. But BCSIL mutations are also described as a cause of Leigh syndrome [6] and impaired complex III assembly with isolated mitochondrial encephalopathy [8].

Here, we report a novel homozygous mutation in the $B C S 1 L$ gene in three patients from two distinct consanguineous Turkish families. All patients displayed severe transitory neonatal metabolic acidosis and persistent renal de Toni-Debré-Fanconi syndrome and hypoglycaemia, followed by a chronic clinical progression characterised by short stature with bone deformities, deafness, mild intellectual disability with microcephaly and chronic renal failure. 


\section{Material and methods}

\section{Biopsy and fibroblast cultures}

Muscle tissue was obtained from the quadriceps by surgical biopsy and a fibroblast culture was established from skin obtained at the muscle biopsy site.

\section{Molecular genetic analysis}

Total DNA was extracted from EDTA-stabilised blood, skin fibroblast culture or kidney using the Qiagen Mini Blood/ Tissue extraction kit following the manufacturer's instructions. MtDNA was screened for deletions using long-template polymerase chain reaction (PCR) as described [17]. MT-CYB, the only mitochondrially encoded complex III subunit was sequenced first. Subsequently, all exons of $B C S 1 L$ were sequenced including the exon/intron boundaries (see supplementary for primer sequences). All PCR fragments were sequenced using BigDye chemistry1.1 on an ABI3100 genetic analyser.

\section{OXPHOS assays and western blotting}

Measurements of skeletal muscle homogenates and fibroblasts were performed as described previously [16]. Individual respiratory chain complex activities and the mitochondrial matrix enzyme citrate synthase (CS) were measured spectrophotometrically in a UV-1601 (Shimadzu) in 1-mL sample cuvettes maintained at $30{ }^{\circ} \mathrm{C}$ [24]. All values are expressed relative to the mitochondrial maker enzyme CS ( $\mathrm{mU} / \mathrm{mU} \mathrm{CS}$ ). Analysis of mitochondrial proteins was performed from skeletal muscle homogenates and isolated mitochondria from fibroblasts separated by 1D blue native gel electrophoresis as described [16]. Subsequent western blotting analysis was performed with antibodies against the core 1 protein of complex III (UQCRC1, MS303 MitoSicence), the Rieske Fe/S protein (UQCRFS1, MS305, MitoScience) and the loading control SDHA (MS204, MitoScience). For 2D analysis, the blue native polyacrylamide gel electrophoresis (BN-PAGE) gel strip was separated on $10 \%$ Tricine/sodium dodecyl sulphate (SDS)-PAGE followed by silver staining. SDS-PAGE was performed after Lämmli [19]. Protein concentrations were determined by BCA methods and equal amounts loaded.

\section{Patients' medical reports}

\section{Patients 1 and 2}

Patient 1 is a 20-year-old male and the second child of healthy parents, who were not known to be consanguineous but came from the same village in Turkey. He was born at term after an uneventful pregnancy. At $20 \mathrm{~h}$ of life, he presented with acute respiratory distress syndrome associated with severe lactic acidosis, elevation of transaminases, hypoglycaemia, and elevated urinary excretion of lactate and Krebs cycle metabolites. The blood results were as follows: lactate $18.7 \mathrm{mmol} / \mathrm{L}$, increased anion gap $35 \mathrm{mmol} / \mathrm{L}$, pH 7.135, $\mathrm{pCO}_{2} 15.2 \mathrm{mmHg}$, $\mathrm{pO}_{2} 120.2 \mathrm{mmHg}, \mathrm{HCO}_{3} 4.9 \mathrm{mmol} / \mathrm{L}$, aspartate aminotransferase (ASAT) $107 \mathrm{U} / \mathrm{L}$ (normal range 11-47 U/L) and alanine aminotransferase (ALAT) $34 \mathrm{U} / \mathrm{L}$ (normal range 7-24 U/L). He also presented marked tubulopathy of Fanconi type with aminoaciduria, phosphaturia, hyperuricosuria and glycosuria. A Fanconi-Bickel syndrome was suspected and ruled out by biochemical and molecular investigations. In contrast to the tubulopathy, the severe neonatal lactic acidosis remitted spontaneously after the first months of life. At the age of 3 years, speech delay was noticed and bilateral sensorineural hearing loss was diagnosed (hearing level was $60 \mathrm{~dB}$ on the right side, $70 \mathrm{~dB}$ on the left) and treated by hearing aids. Despite adequate vitamin $\mathrm{D}$, calcium and phosphate supplementation, genu valgum deformity progressed (Fig. 1a), and at 15 and 20 years, the patient underwent surgical treatment (Fig. 1b, c). The renal function measured by inulin clearance was also progressively compromised with a glomerular filtration rate (mGFR) of $75 \mathrm{~mL} / \mathrm{min}$ per $1.73 \mathrm{~m}^{2}$ and $65 \mathrm{~mL} / \mathrm{min}$ per $1.73 \mathrm{~m}^{2}$ at the ages of 13 and 16 years, respectively. Renal biopsy at this age showed mild lesions compatible with focal segmental glomerulosclerosis and interstitial fibrosis, but with no tubular mitochondrial anomalies at electron microscopic level. He also developed pili torti at the upper arm and face skin. Twice during infancy, normal ferritin levels were measured. At the age of 14 years, his growth parameters were $<3$ rd percentile for both head circumference and height $(144 \mathrm{~cm})$ relative to Turkish growth charts. At 15 years of age, the patient attended a special school. Cerebral magnetic resonance imaging (MRI) and ophthalmologic and cardiac investigations were normal. Mitochondrial complex I-V enzymatic activity was normal in skin fibroblasts. Sequence analysis of the entire mitochondrial genome excluded a pathogenic mtDNA mutation. A muscle biopsy performed 3 years later revealed an isolated complex III deficiency. Coenzyme Q10, carnitine and folinic acid were introduced. At the age of 17 years, his stature was $160.4 \mathrm{~cm}(-2.28 \mathrm{SD})$. Because of pubertal delay and chronic renal failure, a treatment with growth hormone was started and administered for 3 years. The patient is now 20 years old and measured $166.6 \mathrm{~cm}(-1.32 \mathrm{SD})$. At the last control, blood pressure was $114 / 67 \mathrm{mmHg}$. The blood results were as follows: bicarbonate $21.5 \mathrm{mmol} / \mathrm{L}$, lactate $0.77 \mathrm{mmol} /$ $\mathrm{L}$, sodium $141 \mathrm{mmol} / \mathrm{L}$, potassium $3.5 \mathrm{mmol} / \mathrm{L}$, chloride $111 \mathrm{mmol} / \mathrm{L}$, calcium $2.26 \mathrm{mmol} / \mathrm{L}$ and phosphate $0.61 \mathrm{mmol} / \mathrm{L}$. Creatinine was $115 \mu \mathrm{mol} / \mathrm{L}$, with an estimated glomerular filtration rate of $78 \mathrm{~mL} / \mathrm{min} / 1.73 \mathrm{~m}^{2}$ (CKD-EPI, Chronic Kidney Disease-Epidemiology Collaboration formula). Urine analysis disclosed an alkaline urine, glycosuria and mild proteinuria compatible with de Toni-Debré-Fanconi 
Fig 1 Presentation of bone phenotype of patients $1(\mathbf{a}-\mathbf{c})$ and 2 (d). Bone structure and genu valgum of patient 1 at 15 years of age (a) and at 17 years of age (b) showing progressive deformation despite dietary supplementation. (c) Corrective surgery at 20 years of age. (d) Patient 2 (first cousin of patient 1 ) at 17 years of age displaying severe bone deformities and osteopenia

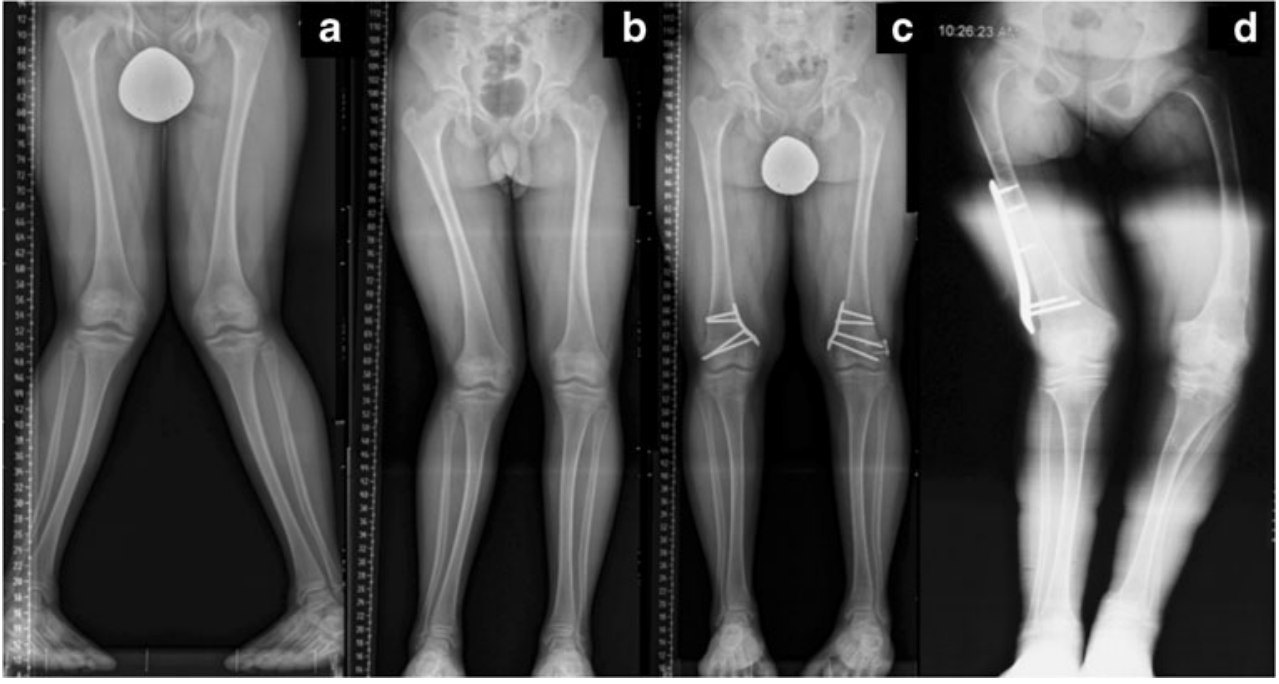

syndrome. On ultrasound imaging, the length of the right kidney was $9.7 \mathrm{~cm}(<\mathrm{P} 5)$, and that of the left kidney $10.6 \mathrm{~cm}$ (P30). There was no sign of nephrocalcinosis but a right kidney stone. Acylcarnitines measured at the age of 5 years were suggestive of a $\beta$-oxidation defect, but were, however, normal in a fasting test in later years. Liver function test was in the normal range with no structural changes. A splenomegaly was noted.

His first cousin (patient 2: III:4) is a 14-year-old girl, who presented with growth retardation, renal Fanconi syndrome, bilateral genu valgum, severe hypophosphataemic rickets, deafness (diagnosed at age 13 years) and intellectual disability. At age 17 years, she displayed severe bone deformities and osteopenia (Fig. 1d).

\section{Patient 3 from Heidelberg}

Patient 3 was born after an uneventful pregnancy at term (Apgar, 9/10/10; pH, 7.26; weight, $2620 \mathrm{~g}$; length, $47 \mathrm{~cm}$; $\mathrm{HC}, 34 \mathrm{~cm}$ ) as second child to consanguineous parents of Turkish origin (great-grandmothers are sisters). On the first day of life, the boy presented with hypoglycaemia and meta-

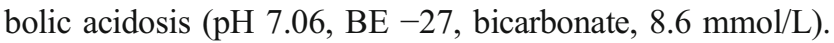
Consequently elevated transaminases were recorded (GOT $80 \mathrm{u} / \mathrm{L}$, GPT $66 \mathrm{u} / \mathrm{L}$ ). Despite resection of a liver cyst at 3 months of age, elevated transaminases persisted. Repeated ultrasounds of the liver and an abdominal MRI, however, were normal. During infancy, a developmental delay was apparent. At 2 years of age, first febrile and later afebrile convulsions occurred that were treated successfully with anticonvulsive therapy. Subsequent electroencephalography revealed primary focal frontotemporal right convulsions with secondary generalisation throughout the brain. Current anticonvulsive therapy includes a daily regimen of levetiracetam $(2 \times 500 \mathrm{mg})$ and oxcarbazepine $(2 \times 600 \mathrm{mg})$. Two cerebral MRIs during the course were unremarkable.
At 3 years of age, sensorineural hearing loss was diagnosed and the boy received hearing aids. At 4 years, during the course of gastroenteritis, the child presented with a fullspectrum Fanconi syndrome with renal-tubular dysfunction and glucosuria, phosphaturia and bicarbonate leak. Consequent hypophosphataemic rickets improved under phosphate substitution. At the age of 7 years, blood lactate was in the normal range with $1.2 \mathrm{mmol} / \mathrm{L}$ (range $0.91-1.75$ ). An abnormal urinary lactate-creatinine ratio of $4 \mathrm{~mol} / \mathrm{mol}$ (range 0-0.15) persisted.

At 9 years of age, he showed short stature (length $118 \mathrm{~cm}$, P3) and microcephaly (head circumference $49.5 \mathrm{~cm},<\mathrm{P} 3$ ). Liver was palpable $2-3 \mathrm{~cm}$ below the costal ridge. Heart and lung were auscultatory unremarkable. Genu varum was improving. Neurological examination shows mild retardation, sensorineural hearing loss and slight ptosis of the left eye, and a mild muscular hypotonia, but was otherwise normal. The patient attended a special school with supportive speech therapy.

\section{Results}

\section{Respiratory activity measurements and complex assembly}

Enzymatic activity for individual respiratory chain (RC) complexes was assessed in isolated mitochondria from skin fibroblasts and in muscle homogenates of patient 1 . Normal activity of the RC complexes was found in fibroblasts. Normal mitochondrial function was further confirmed by oxygen consumption studies in fibroblasts (data not shown). Measurements in muscle homogenate, however, showed an isolated complex III deficiency with a residual activity of $52 \%$ (Table 1 ). Biochemical analysis of skeletal muscle in patient 3 was restrained to combined $\mathrm{CII}+\mathrm{CIII}$ measurement due to limited availability of material revealing residual activity of $38 \%$ (Table 1). 
Table 1 Respiratory chain enzyme activities in isolated mitochondria from cultured skin fibroblasts and in muscle homogenates in patients 1 and 3

\begin{tabular}{|c|c|c|c|c|c|c|}
\hline \multicolumn{7}{|l|}{ Patient 1} \\
\hline Fibroblasts & $\mathrm{CS}^{\mathrm{a}}$ & $\mathrm{CI} / \mathrm{CS}$ & $\mathrm{CII} / \mathrm{CS}$ & $\mathrm{CIII} / \mathrm{CS}$ & $\mathrm{CIV} / \mathrm{CS}$ & $\mathrm{CV} / \mathrm{CS}$ \\
\hline Patient & 202 & 0.27 & 0.31 & 0.66 & 0.79 & 0.13 \\
\hline Reference range & $134-228$ & $0.19-0.46$ & $0.17-0.52$ & $0.35-0.87$ & $0.42-1.11$ & $0.12-0.38$ \\
\hline Muscle & $\mathrm{CS}^{\mathrm{a}}$ & $\mathrm{CI} / \mathrm{CS}$ & $\mathrm{CII} / \mathrm{CS}$ & $\mathrm{CIII} / \mathrm{CS}$ & $\mathrm{CIV} / \mathrm{CS}$ & $\mathrm{CV} / \mathrm{CS}$ \\
\hline Patient & 121 & 0.22 & 0.30 & $0.26(52 \%)$ & 1.00 & 0.44 \\
\hline Reference range & $70-173$ & $0.14-0.28$ & $0.14-0.36$ & $0.50-1.11$ & $0.57-1.76$ & $0.17-0.66$ \\
\hline \multicolumn{7}{|l|}{ Patient 3} \\
\hline Muscle & $\mathrm{CS}^{\mathrm{b}}$ & $\mathrm{CII}+\mathrm{CIII} / \mathrm{CS}$ & & & & \\
\hline Patient & 204 & $0.04(38 \%)$ & & & & \\
\hline Reference range & $(48-110)$ & $0.15-0.34$ & & & & \\
\hline
\end{tabular}

Values in parentheses present the activities as a percentage of the lowest value of control range. OXPHOS complex measurements including complex III in patient-derived fibroblasts were normal with slightly elevated CS. Isolated complex III deficiency with a residual activity of $52 \%$ was detected in skeletal muscle of patient 1 . Combined measurement of $\mathrm{CII}+\mathrm{CIII}$ in skeletal muscle of patient 3 showed residual activity of $38 \%$. CI-V activities are given as $\mathrm{mU} / \mathrm{mU} \mathrm{CS}$. $\mathrm{CII}+\mathrm{CIII}$ activities are given as $\mathrm{mU} / \mathrm{mU} \mathrm{CS}$

${ }^{\text {a }} \mathrm{CS}$ activity ( $\mathrm{mU} / \mathrm{mg}$ homogenate protein)

${ }^{\mathrm{b}} \mathrm{CS}$ activity (mU/mg non-collagen protein)
1D BN-PAGE of skeletal muscle homogenate of patient 1 with subsequent western blotting against the core 1 protein of complex III (UQCRC1) showed a shift in electrophoretic mobility of complex III ( $440 \mathrm{kDa})$ in skeletal muscle compared to controls (Fig. 2a). The supercomplex assembly was comparable to the control. Blotting against the Rieske Fe/S protein UQCRFS1 revealed decreased amounts of assembled BCS1L complex, which was not as prominent in fibroblasts (Fig. 2a). Further analysis by SDS-PAGE showed drastically decreased amounts of UQCRFS1 in the skeletal muscle of the patient, which could also evidenced in isolated fibroblast mitochondria (Fig. 2b). Further separation into the subunits of the individual RC complexes by $2 \mathrm{D}$ native/SDS-PAGE was performed (Fig. 2c). Silver staining revealed equal protein amounts of the core subunits UQCRC1 and UQCRC2 of complex III. In the range of $25-45 \mathrm{kDa}$ of the respiratory subunits (MT-CYB, CYC1 and UQCRFS1), a prominent spot around molecular weight $25 \mathrm{kDa}$ was absent in the patient most likely representing the Rieske Fe/S protein (UQCRFS1) (Fig. 2b, red circle).

\section{Molecular genetic analysis and phylogenetic properties}

Large re-arrangements of the mitochondrial DNA and specific mutation screening were excluded by long-range PCR and direct sequencing from kidney tissue of patient 1 . As the electrophoretic analysis showed a slight shift in mobility suggesting the absence of a subunit with otherwise normal amounts of complex III and supercomplex or respirasome formation (CIII/CIV), MT-CYB (35 kDA) was the first candidate for sequencing, but revealed no mutation. Further insight into the subcomplexes by 2D native/SDS-PAGE prompted sequencing of BCS1L as an assembly factor for the most likely missing subunit UQCRFS1. A novel homozygous variant localised in the protein sequence that is associated with internal mitochondrial import of BCSIL was detected (Fig. 3c). This variant results in an amino acid change at position 48 (p.M48V) predicted to be deleterious in silico (Fig. 3d) and highly conserved among species (Fig. 3d). Homozygosity for this variant was confirmed in all patients, whereas unaffected family members were determined as healthy heterozygous carriers of the c.142A $>$ G mutation (Fig. 3a, b).

\section{Discussion}

We describe three patients with a transient neonatal acidosis, severe Fanconi-type tubulopathy, short stature, sensorineural deafness, microcephaly, mild mental retardation and elevation of liver enzymes due to the novel mutation p.M48V in BCS1L. The clinical phenotype is between Bjornstad and GRACILE syndrome and the novel variant p.M48V is located in the region of amino acids 35 to 73 , where all so far reported mutations cause isolated complex II deficiency. Neonatal lactic acidosis in primary mitochondrial dysfunction is generally a poor prognostic factor. This report, however, highlights that lactic acidosis might be transient in this disorder with relatively good neurological outcome, which is unusual for a RC disorder. Cognitive outcome was variable and could be linked to some extent to the timing of deafness correction. Although specific treatment options for this disease do not exist, an early treatment of Fanconi-type tubulopathy may partially limit bone deformities and improve quality of life and outcome. The result of the excessive loss of phosphate and calcium as also hydroxylation defects to produce 1,25dihydroxyvitamin D3 caused by the proximal tubulopathy 
a

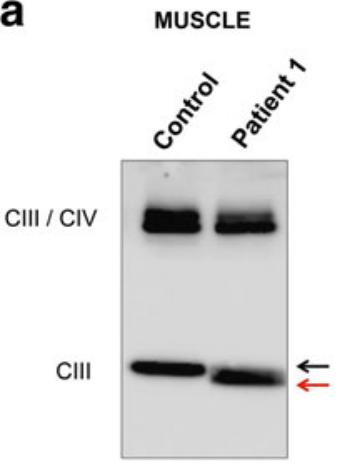

CIII / CIV
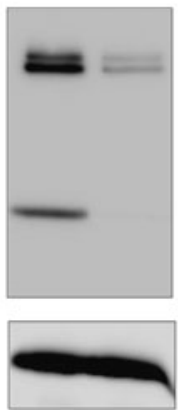

FIBROBLASTS
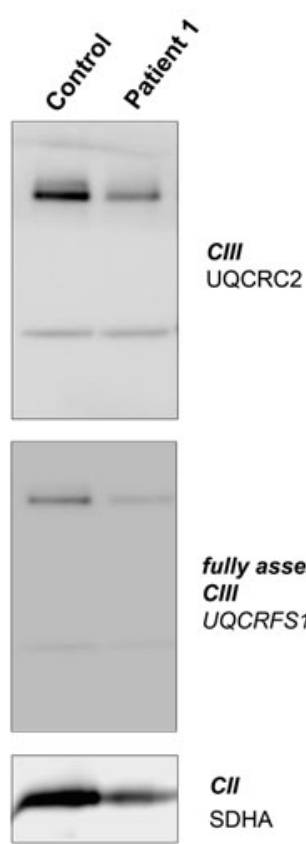

CII SDHA

CIII b
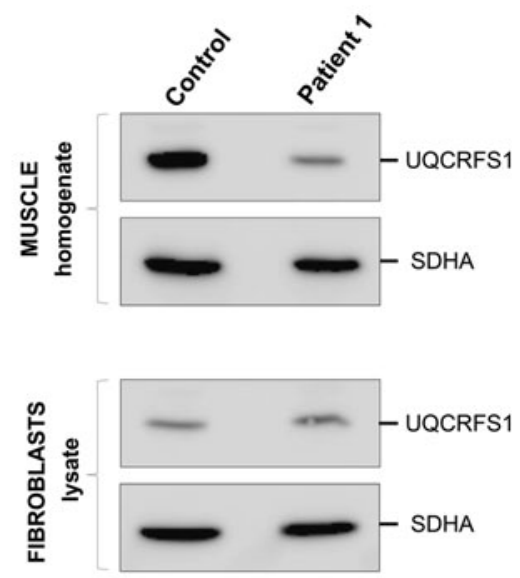

UQCRFS1

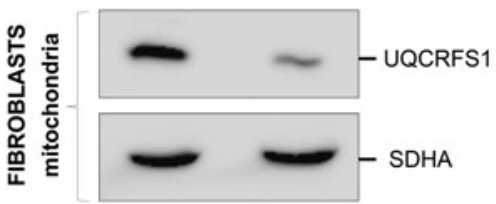

C

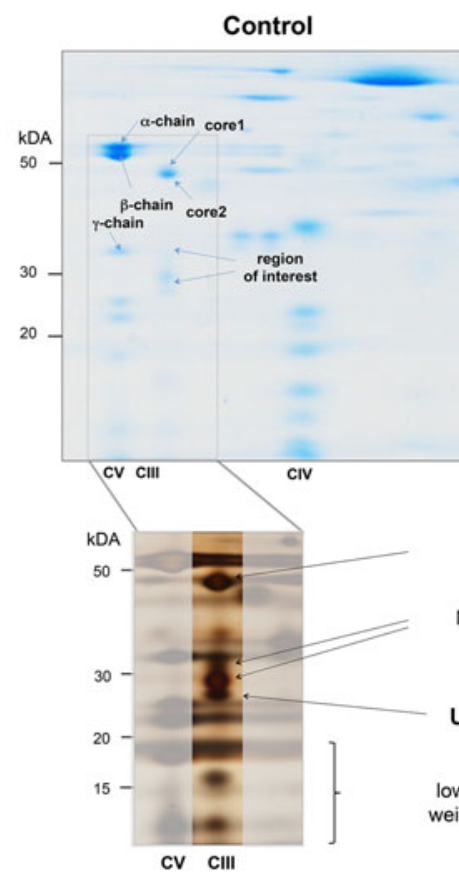

Fig. 2 Analysis of complex III assembly status and localisation of Rieske $\mathrm{Fe} / \mathrm{S}$ protein in skeletal muscle and fibroblasts of patient 1. a 1D BNPAGE western blot analysis of muscle homogenate against the core 1 protein of complex III (UQCRC1) shows a slight mobility shift in the patient's complex III. A diminished amount of the Rieske Fe/S protein (UQCRFS1) in the native complex III in skeletal muscle is evidenced and to a lesser amount in fibroblasts of the patient. b SDS-PAGE reveals a strong decrease of total Rieske Fe/S protein in the patients' skeletal muscle. Comparison of cell lysates and isolated mitochondria from

leads to possible bone deformation as evidenced in our patients. Psychiatric symptoms have been associated with

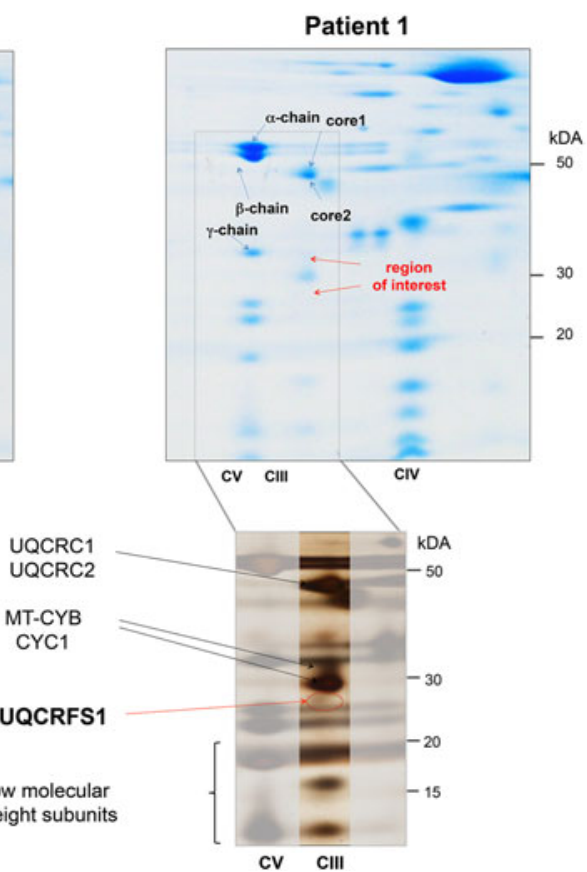

fibroblasts reveal that this decrease of the Rieske $\mathrm{Fe} / \mathrm{S}$ protein is mainly evidenced in the mitochondria. c 2D BN-PAGE of crude muscle homogenate show missing subunit(s) of complex III (right panel) in comparison to control gel (left panel). Detailed analysis by silver staining (lower panels) shows that a spot in the region of the respiratory subunits is missing. This spot most likely represents the Rieske Fe/S protein (UQCRFS1). Known subunits determined from MALDI-TOF experiments are indicated (blue arrows): $\alpha-, \beta$ - and $\gamma$-subunits of complex V; core 1 and core 2 (UQCRC1, UQCRC2)

$B C S 1 L$ mutations as additional clinical feature, but were not present in our patients [1]. 
Fig. 3 Family pedigree (a), molecular genetic analysis (b), gene structure and phylogenetic conservation of BCS1L (c). a The family pedigree of patient 1 (III:1) with affected individuals (III:1, III:4) both being homozygous for the mutation c. $142 \mathrm{~A}>\mathrm{G}$ (left pedigree). The family pedigree of patient 3 (II:1) homozygous for the c. $142 \mathrm{~A}>\mathrm{G}$ with all other family members being healthy heterozygous carriers (right pedigree). $\mathbf{b}$ Sequencing of the $B C S 1 L$ gene revealed a novel homozygous missense mutation at position $142(\mathrm{~A}>\mathrm{G})$ causing a methionine $(\mathrm{M})$ to valine $(\mathrm{V})$ amino acid exchange in both families. c BCS1L (OMIM \#603647) has seven exons (cytogenetic location: 2q23) encoding a chaperone AAA+ ATPase. All reported mutations are marked (HGMD professional built, accessed August, 2015). A basic differentiation into GRACILE (red), isolated complex III deficiency (orange) and Bjornstad syndrome (pink) shows the unpredictability of phenotypic outcome (for review and precise clinical delineation, refer to references $[3,9])$. The novel mutation (boxed) is located within a highly conserved region involved in import of the protein. n.a. not available, TMD

transmembrane domain, MTS mitochondrial targeting sequence, $I A S$ import auxiliary sequence a

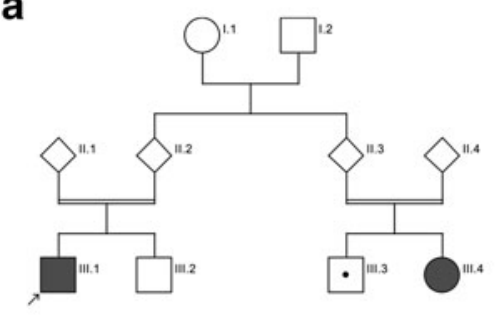

b
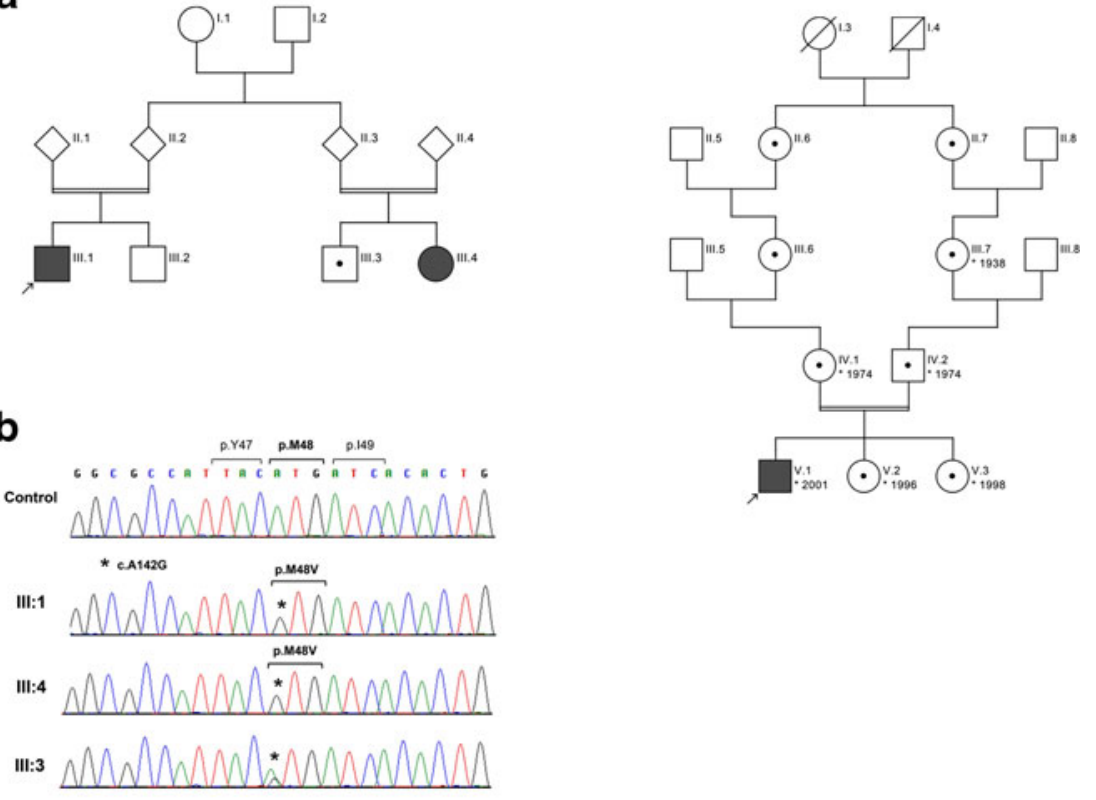

C

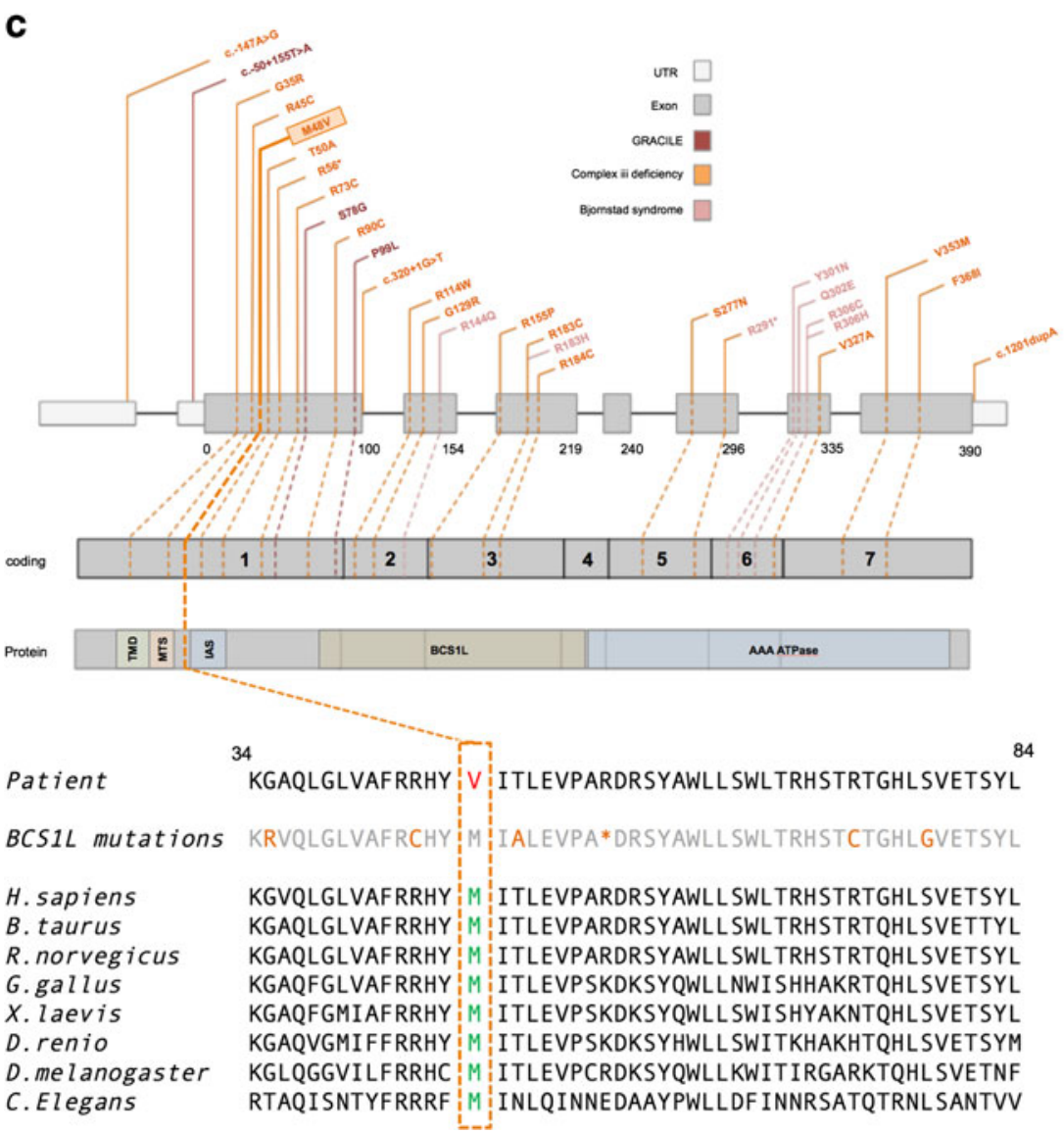

BCS1L mutations are an especially frequent cause of complex III deficiencies in patients of Turkish origin. De Lonlay et al. reported that they were able to detect a $B C S 1 L$ mutation in one third of their patients with complex III deficiency [6]. Interestingly, they also reported that the clinical presentation was always associated with neonatal tubulopathy with hepatic 
involvement and encephalopathy. Hepatic involvement in our patients is therefore likely caused by dysfunctional BCS1L. BCS1L has no common cleavable mitochondrial targeting peptide, but studies have confirmed that the protein is imported into the mitochondria by a conserved domain of the N-terminus (spanning residues 1-89 [8]) responsible for the import and intramitochondrial sorting [22]. The Saccharomyces cerevisiae homolog of human BCS1L has three distinct sequences containing i) an anchoring transmembrane domain (residues 52-68), ii) an internal targeting sequence consisting of an amphipathic $\alpha$-helical structure (residues 69-83), which is iii) adjacent to an auxiliary import region (residues 84-126) for translocation via the TOM complex $[8,10]$. We speculate that this mutation affects the sorting of BCS1L to the mitochondria. In comparison to the now reported p.M48V mutation, the p.T50A mutation shows a quite distinctive phenotype including coarse facial features and abnormal distribution of subcutaneous fat [3]. The wide phenotypic range and presentation in relation to the mutational location therefore remains unclear, but in severity seems to correlate with the complex III-associated amount of ROS production [14]. Furthermore, the BCS1L protein is implicated in distinctive cellular functions, whereas the GRACILE syndrome possibly is related to iron homeostasis and the CIII assembly to OXPHOS deficiency phenotypes [8].

The decreased complex III activity observed in our patients is most likely caused by dysfunctional complex III, although interestingly the supercomplex formation was not impaired. Dysfunctional BCS1L protein impairs the correct integration of the Rieske iron-sulphur protein into complex III [4, 23]. Analysis of the protein composition of complex III in our patient indicated the missing subunit to be the Rieske Fe/S protein as revealed by native 1D BN-PAGE with subsequent western blotting and 2D BN-PAGE with subsequent silver staining. Analysis of native complex III from fibroblasts did not reveal a prominent shift of the complex as seen in skeletal muscle. However, a decrease of the Rieske Fe/S protein in isolated fibroblast mitochondria was revealed, possibly representing degradation or lowered protein stability if not mediated by BCS1L to form the holocomplex.

In contrast to the p.T50A mutation, the p.M48V defect was not detectable in fibroblasts. In literature, tissue-specific effects of BCS1L mutations on complex III activity have been described $[8,20]$. It is suggested that depending on the tissue, even low amounts of complex III are sufficient in the optimised in vitro assays to obtain normal enzymatic activity. This high threshold effect may make it difficult to prove a complex III defect in a given tissue and stresses the importance to evaluate different tissues as in other mitochondrial defects $[5,18]$.

In silico calculations for this variant are predicted to be deleterious and the phylogenetic alignment shows high conservation. Irrespective of location and phenotype, however, impaired complex III (and finally respirasome) assemblyfor currently unknown reasons - results in increased reactive oxygen species (ROS) production, which seems to directly correlate with the clinical outcome [14]. The broad clinical spectrum and unexplained tissue specificity of different BCS1L have to be further elucidated and serve as a model for pathophysiological expression of respiratory chain diseases [2].

Molecular confirmation of mitochondrial diagnosis is often burdensome but very important for the genetic counselling and the care of the families. Accurate molecular diagnosis allowed for prenatal testing, which is especially important in families with a higher or unknown rate of consanguinity (in families with many multiple marriages of first cousins).

Here, we report a novel mutation in the $B C S 1 L$ gene associated with hypoglycaemia, glycosuria, deafness, short stature and rickets, Fanconi syndrome and severe lactic acidosis in the neonatal period in three patients with isolated complex III deficiency born to consanguineous parents. In contrast to other reported cases of BCS1L mutations, our patients did not present with any specific dysmorphic features (e.g. skin fat pads) [3]. Biochemical analysis of fibroblasts of our patients did not reveal a detectable enzymatic CIII defect, which is an unusual finding in contrast to most reported BCS1L mutations. The CIII defect was only apparent in muscle tissue and undetectable in patients' fibroblasts, delaying the specific molecular diagnosis. Strikingly, disease severity and progression of phenotypes caused by mutated BCS1L seem to be unpredictable based on their location within the protein (Fig. 3c). We propose that BCS1L mutation screening should be routinely included in the differential diagnosis of severe glomerular renal insufficiency (proximal tubulopathy) in the newborn period.

Acknowledgments The authors are indebted to the families participating in this study.

Authors' contributions CBJ and JMN drafted the manuscript. Study design was performed by CBJ, LB and JMN. UK has drafted and provided clinical description of patient 3 , and MFB provided biochemical data of patient 3. CJ, DH, AH and SE performed biochemical experimentation. MFB, AS and SG conducted genetic testing. AF, HC, FB, CT and LB conducted and drafted the clinical part of patients 1 and 2 . All authors have reviewed and approved the final manuscript.

\section{Compliance with ethical standards}

Conflict of interest The authors declare that they have no competing interests.

Ethical approval Parental consent has been obtained to submit this report for publication. Informed consent was obtained from all participating individuals and the study was approved by the local ethical committee of the Canton of Bern. 


\section{References}

1. Al-Owain M, Colak D, Albakheet A, Al-Younes B, Al-Humaidi Z, Al-Sayed M, Al-Hindi H, Al-Sugair A, Al-Muhaideb A, Rahbeeni Z, Al-Sehli A, Al-Fadhli F, Ozand PT, Taylor RW, Kaya N (2013) Clinical and biochemical features associated with BCS1L mutation. J Inherit Metab Dis 36:813-820. doi:10.1007/s10545-012-9536-4

2. Bénit P, Lebon S, Rustin P (2009) Respiratory-chain diseases related to complex III deficiency. Biochim Biophys Acta 1793:181185. doi:10.1016/j.bbamcr.2008.06.004

3. Blázquez A, Gil-Borlado MC, Morán M, Verdú A, Cazorla-Calleja MR, Martín MA, Arenas J, Ugalde C (2009) Infantile mitochondrial encephalomyopathy with unusual phenotype caused by a novel BCS1L mutation in an isolated complex III-deficient patient. Neuromuscul Disord 19:143-146. doi:10.1016/j.nmd.2008.11.016

4. Conte L, Zara V (2011) The Rieske iron-sulfur protein: import and assembly into the cytochrome bc(1) complex of yeast mitochondria. Bioinorg Chem Appl 2011:363941-363949. doi:10.1155/ 2011/363941

5. Davey GP, Canevari L, Clark JB (1997) Threshold effects in synaptosomal and nonsynaptic mitochondria from hippocampal CA1 and paramedian neocortex brain regions. J Neurochem 69:25642570

6. de Lonlay P, Valnot I, Barrientos A, Gorbatyuk M, Tzagoloff A, Taanman JW, Benayoun E, Chrétien D, Kadhom N, Lombès A, de Baulny HO, Niaudet P, Munnich A, Rustin P, Rotig A (2001) A mutant mitochondrial respiratory chain assembly protein causes complex III deficiency in patients with tubulopathy, encephalopathy and liver failure. Nat Genet 29:57-60. doi:10.1038/ng706

7. Fellman V, Rapola J, Pihko H, Varilo T, Raivio KO (1998) Ironoverload disease in infants involving fetal growth retardation, lactic acidosis, liver haemosiderosis, and aminoaciduria. Lancet 351:490 493. doi:10.1016/S0140-6736(97)09272-6

8. Fernandez-Vizarra E, Bugiani M, Goffrini P, Carrara F, Farina L, Procopio E, Donati A, Uziel G, Ferrero I, Zeviani M (2007) Impaired complex III assembly associated with BCS1L gene mutations in isolated mitochondrial encephalopathy. Hum Mol Genet 16:1241-1252. doi:10.1093/hmg/ddm072

9. Fernandez-Vizarra E, Zeviani M (2015) Nuclear gene mutations as the cause of mitochondrial complex III deficiency. Front Genet. doi: 10.3389/fgene. 2015.00134

10. Fölsch H, Guiard B, Neupert W, Stuart RA (1996) Internal targeting signal of the BCS1 protein: a novel mechanism of import into mitochondria. EMBO J 15:479-487

11. Ghezzi D, Arzuffi P, Zordan M, Da Re C, Lamperti C, Benna C, D'Adamo P, Diodato D, Costa R, Mariotti C, Uziel G, Smiderle C, Zeviani M (2011) Mutations in TTC19 cause mitochondrial complex III deficiency and neurological impairment in humans and flies. Nat Genet 43:259-263. doi:10.1038/ng.761

12. Ghezzi D, Zeviani M (2012) Assembly factors of human mitochondrial respiratory chain complexes: physiology and pathophysiology. Adv Exp Med Biol 748:65-106. doi:10.1007/978-1-4614-3573-0_4

13. Hinson JT, Fantin VR, Schönberger J, Breivik N, Siem G, McDonough B, Sharma P, Keogh I, Godinho R, Santos F, Esparza A, Nicolau Y, Selvaag E, Cohen BH, Hoppel CL, Tranebjaerg L, Eavey RD, Seidman JG, Seidman CE (2007) Missense mutations in the BCS1L gene as a cause of the Björnstad syndrome. N Engl J Med 356:809-819. doi:10.1056/ NEJMoa055262

14. Hinson JT, Fantin VR, Schönberger J, Breivik N, Siem G, McDonough B, Sharma P, Keogh I, Godinho R, Santos F, Esparza A, Nicolau Y, Selvaag E, Cohen BH, Hoppel CL, Tranebjaerg L, Eavey RD, Seidman JG, Seidman CE (2007) Missense mutations in the BCS1L gene as a cause of the Björnstad syndrome. N Engl J Med 356:809-819. doi:10.1056/NEJMoa055262
15. Invernizzi F, Tigano M, Dallabona C, Donnini C, Ferrero I, Cremonte M, Ghezzi D, Lamperti C, Zeviani M (2013) A homozygous mutation in LYRM7/MZM1L associated with early onset encephalopathy, lactic acidosis, and severe reduction of mitochondrial complex III activity. Hum Mutat 34:1619-1622. doi:10.1002/ humu. 22441

16. Jackson CB, Nuoffer JM, Hahn D, Prokisch H, Haberberger B, Gautschi M, Haberli A, Gallati S, Schaller A (2014) Mutations in SDHD lead to autosomal recessive encephalomyopathy and isolated mitochondrial complex II deficiency. J Med Genet 51:170-175. doi:10.1136/jmedgenet-2013-101932

17. Kleinle S, Wiesmann U, Superti-Furga A, Krähenbühl S, Boltshauser E, Reichen J, Liechti-Gallati S (1997) Detection and characterization of mitochondrial DNA rearrangements in Pearson and Kearns-Sayre syndromes by long PCR. Hum Genet 100:643-650

18. Kotarsky H, Karikoski R, Mörgelin M, Marjavaara S, Bergman P, Zhang D-L, Smet J, van Coster R, Fellman V (2010) Characterization of complex III deficiency and liver dysfunction in GRACILE syndrome caused by a BCS1L mutation. Mitochondrion 10:497-509. doi:10.1016/j.mito.2010.05.009

19. Laemmli UK (1970) Cleavage of structural proteins during the assembly of the head of bacteriophage T4. Nature 227:680-685

20. Marín-Buera L, García-Bartolomé A, Morán M, López-Bernardo E, Cadenas S, Hidalgo B, Sánchez R, Seneca S, Arenas J, Martín MA, Ugalde C (2014) Differential proteomic profiling unveils new molecular mechanisms associated with mitochondrial complex III deficiency. J Proteome 113C:38-56. doi:10.1016/j.jprot.2014.09.007

21. McFarland R, Turnbull DM (2009) Batteries not included: diagnosis and management of mitochondrial disease. J Intern Med 265: 210-228. doi:10.1111/j.1365-2796.2008.02066.x

22. Moran (2010) Cellular pathophysiological consequences of BCS1L mutations in mitochondrial complex III enzyme deficiency. 1-12. doi:10.1002/humu.21294

23. Nobrega FG, Nobrega MP, Tzagoloff A (1992) BCS1, a novel gene required for the expression of functional Rieske iron-sulfur protein in Saccharomyces cerevisiae. EMBO J 11:3821-3829

24. Shepherd D, Garland PB (1969) The kinetic properties of citrate synthase from rat liver mitochondria. Biochem J 114: 597-610

25. Suomalainen A (2011) Therapy for mitochondrial disorders: little proof, high research activity, some promise. Semin Fetal Neonatal Med 16:236-240. doi:10.1016/j.siny.2011.05.003

26. Tucker EJ, Wanschers BFJ, Szklarczyk R, Mountford HS, Wijeyeratne XW, van den Brand MAM, Leenders AM, Rodenburg RJ, Reljić B, Compton AG, Frazier AE, Bruno DL, Christodoulou J, Endo H, Ryan MT, Nijtmans LG, Huynen MA, Thorburn DR (2013) Mutations in the UQCC1-interacting protein, UQCC2, cause human complex III deficiency associated with perturbed cytochrome b protein expression. PLoS Genet 9: e1004034. doi:10.1371/journal.pgen.1004034

27. Visapää I, Fellman V, Vesa J, Dasvarma A, Hutton JL, Kumar V, Payne GS, Makarow M, van Coster R, Taylor RW, Turnbull DM, Suomalainen A, Peltonen L (2002) GRACILE syndrome, a lethal metabolic disorder with iron overload, is caused by a point mutation in BCS1L. Am J Hum Genet 71:863-876. doi:10.1086/342773

28. Wagener N, Ackermann M, Funes S, Neupert W (2011) A pathway of protein translocation in mitochondria mediated by the AAAATPase Bcs1. Mol Cell 44:191-202. doi:10.1016/j.molcel.2011. 07.036

29. Wanschers BFJ, Szklarczyk R, van den Brand MAM, Jonckheere A, Suijskens J, Smeets R, Rodenburg RJ, Stephan K, Helland IB, Elkamil A, Rootwelt T, Ott M, van den Heuvel L, Nijtmans LG, Huynen MA (2014) A mutation in the human CBP4 ortholog UQCC3 impairs complex III assembly, activity and cytochrome b stability. Hum Mol Genet. doi:10.1093/hmg/ ddu357 